Available online at http://jddtonline.info

MINI REVIEW

\title{
AN UPDATED REVIEW ON HEPATOPROTECTIVE MEDICINAL PLANTS
}

\author{
Areefa Shaik*, A Elumalai, M Chinna Eswaraiah, Usha \\ Department of Pharmacognosy, Anurag Pharmacy College, Ananthagiri (v), Kodad (M), Nalgonda (DT), Andhra Pradesh, India, 508 \\ 206. \\ Corresponding author's Email: areefa07@gmail.com
}

Received 29 Jan 2011; Revised 06 Feb 2012; Accepted 02 March 2012, Available online 15 March 2012

\begin{abstract}
Medicinal plants may serve as a vital source of potentially useful new compounds for the development of effective therapy to combat a variety of liver problems. Many herbs have been proven to be effectual as hepatoprotective agents while many more are claimed to be hepatoprotective but lack any such scientific evidence to support such claims. Developing a satisfactory herbal therapy to treat severe liver diseases requires systematic investigation of properties like anti-hepatotoxicity (antioxidants), stimulation of liver regeneration and choleretic activity. Formulation of herbal medicines with standards of safety and efficacy can revitalize treatment of liver disorders. The aim of this review is to elucidate the list of hepatoprotective medicinal plants, which are scientifically proved during jan-dec 2011.
\end{abstract}

Key Words: Medicinal plants, hepatoprotective agents, treatment of liver disorders.

\section{INTRODUCTION}

Medicinal plants play a key role in the human health care. About $80 \%$ of the world population relies on the use of traditional medicine which is predominantly based on plant materials ${ }^{1}$. The traditional medicine refers to a broad range of ancient natural health care practices including folk/tribal practices as well as Ayurveda, Siddha and Unani. These medical practices originated from time immemorial and developed gradually, to a large extent, by relying or based on practical experiences without significant references to modern scientific principles.

It is estimated that about 7,500 plants are used in local health traditions in, mostly, rural and tribal villages of India. Out of these, the real medicinal value of over 4,000 plants is either little known or hitherto unknown to the mainstream population. The classical systems of medicine such as Ayurveda, Siddha, Amchi, Unani and Tibetan use about 1,200 plants $^{2}$. A detailed investigation and documentation of plants used in local health traditions and pharmacological evaluation of these plants and their taxonomical relatives can lead to the development of invaluable plant drugs for many dreaded diseases. Random screening of plants has not proved economically effective ${ }^{3}$.

Liver damage is very common since liver has to detoxicate lot many toxic substances. Most of the hepatotoxic chemicals damage liver cells primarily by producing reactive species which form covalent bond with the lipids of the tissue. Due to excessive exposure to hazardous chemicals, sometimes the free radicals generated are so high that they overpower the natural defensive system leading to hepatic damage and cause jaundice, cirrhosis and fatty liver. Production of the reactive species depletion manifests in tissue thiol depletion, lipid peroxidation, plasma membrane damage etc., culminating into severe hepatic injury ${ }^{4}$.

\section{CONCLUSION}

From this study, it is clear that the medicinal plants play a vital role against on various diseases. Various herbal plants and plants extracts have significant hepatoprotective activity in animal models. The hepatoprotective activity is probably due to the presence of flavonoids in all few herbal plants. The results of this study indicate that extracts of leaves and plants extracts of some medicinal plant have good potentials for use in hepatic disease. The present review study give evidential explore mechanism of action of medicinal plants against experimentally induced hepatotoxicity. Hence the review study is concluded that the herbal drug possesses hepatoprotective activity and it has been proved by different animal models give many links to develop the future trials. 
Table 1: List of Hepatoprotective plants

\begin{tabular}{|c|c|c|c|c|c|c|}
\hline Botanical name & Family & Parts used & $\begin{array}{c}\text { Solvent } \\
\text { used }\end{array}$ & $\begin{array}{c}\text { Chemical } \\
\text { constituents }\end{array}$ & Screening method & Reference \\
\hline $\begin{array}{l}\text { Amaranthus } \\
\text { caudatus Linn }\end{array}$ & Amaranthaceae & Whole plant & Methanol & $\begin{array}{l}\text { Flavonoids, sapo } \\
\text { nins,glycosides }\end{array}$ & $\begin{array}{c}\text { Carbon tetra } \\
\text { chloride induced }\end{array}$ & $\begin{array}{c}\text { Kuruba } \\
\text { lakshman et } \\
\mathrm{al}^{5}\end{array}$ \\
\hline $\begin{array}{c}\text { Anisochilus } \\
\text { carnosus } \\
\text { Linn }\end{array}$ & Lamiaceae & Stems & Ethanol & $\begin{array}{l}\text { Alkaloids, } \\
\text { flavonoids, } \\
\text { glycosides }\end{array}$ & $\begin{array}{c}\text { Carbon tetra } \\
\text { chloride induced }\end{array}$ & $\begin{array}{c}\text { Venkatesh } \\
\text { et al }\end{array}$ \\
\hline $\begin{array}{c}\text { Asparagus } \\
\text { racemosus Linn }\end{array}$ & Asparagaceae & Roots & Ethanol & $\begin{array}{l}\text { Phenols, } \\
\text { coumarins }\end{array}$ & $\begin{array}{l}\text { Paracetamol } \\
\text { induced }\end{array}$ & $\begin{array}{c}\text { Fasalu } \\
\text { rahimom } \\
\text { om et al }^{7}\end{array}$ \\
\hline Azima tetracantha & Salvadoracaeae & Leaves & Ethanol & $\begin{array}{l}\text { Flavonoids, } \\
\text { triterpenoids }\end{array}$ & $\begin{array}{l}\text { Paracetamol } \\
\text { induced }\end{array}$ & $\begin{array}{c}\text { Arthika et } \\
\mathrm{al}^{8}\end{array}$ \\
\hline $\begin{array}{c}\text { Calotropis procera } \\
\text { R.Br }\end{array}$ & Asclepediaceae & Root bark & Methanol & $\begin{array}{l}\text { Terpinoidsglyco } \\
\text { sides,flavonoids }\end{array}$ & $\begin{array}{c}\text { Carbon tetra } \\
\text { chloride induced }\end{array}$ & $\begin{array}{c}\text { Pati prakash } \\
\text { et al }\end{array}$ \\
\hline $\begin{array}{l}\text { Cajanus cajan } \\
\text { Linn }\end{array}$ & Leguminosae & $\begin{array}{l}\text { Pigeon pea } \\
\text { leaf }\end{array}$ & ethanol & $\begin{array}{l}\text { Flavonoids, } \\
\text { stibenes }\end{array}$ & D-galactosamine & $\begin{array}{c}\text { Oluseye ade } \\
\text { boye } \\
\text { akinloye et } \\
\text { al }^{10}\end{array}$ \\
\hline $\begin{array}{c}\text { Cajanus } \\
\text { scarabaeoides } \\
\text { Linn }\end{array}$ & Fabaeceae & Whole plant & $\begin{array}{c}\text { n- } \\
\text { butanol,etha } \\
\text { nol }\end{array}$ & Flavonoids & $\begin{array}{l}\text { Paracetamol } \\
\text { induced }\end{array}$ & $\begin{array}{c}\text { Suman } \\
\text { pattanayak } \\
\text { et al }^{11}\end{array}$ \\
\hline $\begin{array}{l}\text { Carissa carindas } \\
\text { Linn }\end{array}$ & Apocyanaceae & Root & Ethanol & $\begin{array}{c}\text { Alkaloids, } \\
\text { tannins, steroids }\end{array}$ & $\begin{array}{c}\text { Carbon tetra } \\
\text { chloride induced }\end{array}$ & $\begin{array}{l}\text { Balkrishnan } \\
\text { et al }^{12}\end{array}$ \\
\hline $\begin{array}{l}\text { Clitoria ternatea } \\
\text { Linn }\end{array}$ & Fabaceae & Leaves & Methanol & $\begin{array}{c}\text { Phenolic } \\
\text { flavonoids }\end{array}$ & $\begin{array}{c}\text { Paracetamol } \\
\text { induced }\end{array}$ & $\begin{array}{l}\text { Yeng chen } \\
\text { et al }^{13}\end{array}$ \\
\hline $\begin{array}{c}\text { Cucumis trigonus } \\
\text { Roxb }\end{array}$ & Cucurbitaceae & Fruit & $\begin{array}{l}\text { Pet.ether, } \\
\text { chloroform, } \\
\text { alcohol, } \\
\text { aqueous }\end{array}$ & Flavonoids & $\begin{array}{c}\text { Carbon tetra } \\
\text { chloride induced }\end{array}$ & $\begin{array}{c}\text { Mohammad } \\
\text { imtiaz et } \\
\mathrm{al}^{14}\end{array}$ \\
\hline $\begin{array}{c}\text { Ficus religiosa } \\
\text { Linn }\end{array}$ & Moraceae & Stem bark & Methanol & $\begin{array}{c}\text { Glycosides, } \\
\text { steroids, tannins }\end{array}$ & $\begin{array}{c}\text { Paracetamol } \\
\text { induced }\end{array}$ & $\begin{array}{c}\text { Kavitha } \\
\text { suryawanshi } \\
\text { et al }^{15}\end{array}$ \\
\hline $\begin{array}{l}\text { Garcinia indica } \\
\text { Linn }\end{array}$ & Clusiaceae & Fruit rind & Ethanol & $\begin{array}{l}\text { benzophenones, } \\
\text { garcinol }\end{array}$ & $\begin{array}{c}\text { Carbon tetra } \\
\text { chloride induced }\end{array}$ & $\begin{array}{c}\text { Amol } \\
\text { Bhalchandra } \\
\text { Deore et al }^{16}\end{array}$ \\
\hline $\begin{array}{l}\text { Gmelina asiatica } \\
\text { Linn }\end{array}$ & Verbenaceae & Aerial parts & $\begin{array}{l}\text { Ethanol, } \\
\text { chloroform }\end{array}$ & Flavonoids & $\begin{array}{l}\text { Carbon tetra } \\
\text { chloride }\end{array}$ & $\begin{array}{c}\text { Partha } \\
\text { sarathy et } \\
\text { al }^{17}\end{array}$ \\
\hline $\begin{array}{l}\text { Hyptis suaveolens } \\
\text { linn }\end{array}$ & Lamiaceae & leaves & Aqueous & Flavonoids & $\begin{array}{c}\text { Acetaminophen } \\
\text { induced }\end{array}$ & $\begin{array}{c}\text { Babalola et } \\
\text { al }^{18}\end{array}$ \\
\hline Leucas cilita Linn & Lamiaceae & Whole plant & Ethanol & Flavonoids & $\begin{array}{c}\text { Carbon tetrachloride } \\
\text { induced }\end{array}$ & $\begin{array}{c}\text { Qureshi et } \\
\mathrm{al}^{19}\end{array}$ \\
\hline $\begin{array}{l}\text { Melia azhadirecta } \\
\text { Linn }\end{array}$ & piperaceae & leaves & ethanol & $\begin{array}{l}\text { Spectro photo } \\
\text { metric method }\end{array}$ & $\begin{array}{c}\text { Carbon tetra } \\
\text { chloride,silymarin }\end{array}$ & $\begin{array}{l}\text { H.rajeswary } \\
\text { et al }^{20}\end{array}$ \\
\hline $\begin{array}{c}\text { Morinda citrifolia } \\
\text { Linn }\end{array}$ & Rubiaceae & Fruit & Aqueous & $\begin{array}{c}\text { Saponins,tri } \\
\text { terpins,steroids }\end{array}$ & $\begin{array}{l}\text { Streptozotocin } \\
\text { induced }\end{array}$ & 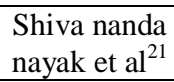 \\
\hline $\begin{array}{c}\text { Myoporum lactum } \\
\text { Linn }\end{array}$ & Myoporaceae & Leaves & $\begin{array}{l}\text { Methanol,n- } \\
\text { butanol }\end{array}$ & Flavonoids & Profenofos induced & $\begin{array}{c}\text { mohammad } \\
{\text { et } \mathrm{al}^{22}}^{22}\end{array}$ \\
\hline $\begin{array}{c}\text { Myrtus communis } \\
\text { Linn }\end{array}$ & Myrtaceae & Leaves & Silymarin & $\begin{array}{c}\text { Flavonoids, } \\
\text { terpenoids, } \\
\text { steroids } \\
\end{array}$ & $\begin{array}{l}\text { Paracetamol } \\
\text { induced }\end{array}$ & $\begin{array}{c}\text { Pasumarthi } \\
\text { phaneendra } \\
\text { et al }^{23}\end{array}$ \\
\hline $\begin{array}{c}\text { Solanum nigram } \\
\text { Linn }\end{array}$ & Solanaceae & Fruits & Ethanol & $\begin{array}{l}\text { Flavonoids, } \\
\text { terpenoids }\end{array}$ & $\begin{array}{l}\text { Carbon tetra } \\
\text { chloride }\end{array}$ & $\begin{array}{c}\text { Subash et } \\
\mathrm{al}^{24}\end{array}$ \\
\hline
\end{tabular}

\section{ACKNOWLEDGEMENT}

Authors are grateful thanks to Anurag Pharmacy College for carrying out this review work. 


\section{REFERENCE}

1. WHO, Regional Office For The Western Pacific, Research Guidelines For Evaluating The Safety And Efficacy Of Herbal Medicines, Manila, WHO, 1993.

2. Pushpangadan P. Role of Traditional Medicine in Primary Health Care. In: Iyengar PK, Damodaran VK, Pushpangadan P, Editors. Science for Health. Published By State Committee On Science, Technology And Environment, Govt. Of Kerala, 1995.

3. Aszalos A, Editor. Antitumor Compounds of Natural Origin. Boca Raton, CRC Press, 1982.

4. Gupta Amartya K, Ganguly Partha, Majumder Upal K, Ghosal Shibnath, Hepatoprotective \& antioxidant effect \& stereoidal saponins of solanum of Solanum xanthocarpum \& Solanum nigrum in paracetomol induce hepatotoxicity in rats, Pharmacologyonline 2009, 1, 757-768.

5. Bagepalli Srinivas, Ashok Kumar, Kuruba Lakshman, Paresandra Avalakondarayppa Arun Kumar, Gollapalle Lakshminarayana Shastry Viswanpha, Veeresh, Prabhakar Veerapur, Boreddy, Shivanadpea, Thippeswamy, Bachappaa,Manoj. Hepatoprotective activity of methanol extracts of Amaranthus caudatus Linn. against paracetamolinduced hepatic injury in rats, Journal of Chinese Integrative Medicine 2011, 9(2), 194-200.

6. Venkatesh P, Dinakar A, Senthilkumar N, Hepatoprotective activity of an ethanolic extract of stems of Anisochilus carnosus against carbon tetrachloride induced hepatotoxicity in rats, International Journal of Pharmacy and Pharmaceutical Sciences 2011, 3(1), 243-245.

7. Fasalu Rahiman, Rupesh Kumar M, Tamizh Mani T, Mohamed Niyas K, Satya Kumar B, Phaneendra P, Surendra B, Hepatoprotective activity of "Asparagus racemosus root" on liver damage caused by paracetamol in rats, Indian Journal of Novel Drug Delivery 2011, 3(2), 112-117.

8. Arthika S, Shanthammal Y, Sheryl Igal1 N, Elankini P, Pramod Reddy G, Gaidhani SN, Ganesan R, Hepatoprotective activity of the ethanolic extract of Azima tetracantha against paracetamol-induced hepatotoxicity in wistar albino rats, Journal of Advances in Pharmacy and Healthcare Research, 2011, 1(2), 14-20.

9. Patil Prakash, Prasad K, Nitin M, Vinay Kumar M, Sreenivasa Rao K, Evaluation of hepatoprotective effect of Calotropis procera $\mathrm{R} . \mathrm{Br}$ root extract against $\mathrm{CCL}_{4}$ induced hepato-oxidative stress in albino rats, International Journal in Ayurveda \& Pharmacy 2011, 2(1), 319-324.

10. Oluseyi Adeboye Akinloye, Moshood Olajire Olaniyi, Hepatoprotective effect of Cajanus cajan on tissue defense system in D-galactosamine-induced hepatitis in rats, Turk $\mathbf{J}$ Biochem 2011, 36(3), 237-241.

11. Suman Pattanayak, Siva Sankar Nayak, Durga Prasad Panda, Subas Chandra Dinda, Vikas Shende, Amol Jadav, Hepatoprotective activity of crude flavonoids extract of Cajanus scarabaeoides (L) in paracetamol intoxicated albino rats, Asian J Pharm Biol Res 2011, 1(1), 22-27.

12. Balakrishanan N, Balasubaramaniam A, Sangameswaran B, Bhaskar VH, Hepatoprotective activity of two Indian medicinal plants from Western Ghats-Tamil nadu, Journal Of Natural Pharmaceuticals 2011, 2( 2), 92-98.
13. Kuppan Nithianantham, Murugesan Shyamala, Yeng Chen, Lachimanan Yoga Latha, Subramanion L. Jothy, Sreenivasan Sasidharan, Hepatoprotective potential of Clitoria ternatea leaf extract against paracetamol induced damage in mice, Molecules 2011, 16, 10134-10145.

14. Kalpana Patila, Shaikh Mohammed Imtiaza, Anoop Singha, Varsha Bagewadia, Shaikh Gazib, Hepatoprotective activity of Cucumis trigonus Roxb. fruit against $\mathrm{CCL}_{4}$ induced hepatic damage in rats, Iranian Journal of Pharmaceutical Research 2011,10(2), 295-299.

15. Kavita Suryawanshi, Sanjay Khakre, Ashish Chourasia, Chaurasiya PK, Pawar RS, Deenanath Jhade, Hepatoprotective activity of stem bark extracts of Ficus religiosa Linn in rats, International Journal of Biomedical Research 2011, 2(8), 466-475.

16. Amol Bhalchandra Deore, Vinayak D Sapakal, Nilofer S Naikwade, Antioxidant and Hepatoprotective activity of Garcinia indica Linn fruit rind, Pharmacie Globale International Journal of Comprehensive Pharmacy 2011, 2(6), 1-5.

17. Merlin NJ and Parthasarathy V, Antioxidant and Hepatoprotective activity of chloroform and ethanol extracts of Gmelina asiatica aerial parts, Journal of Medicinal Plants Research 2011, 5(4), 533-538.

18. Babalola O, Ojo E, Oloyede F, Hepatoprotective activity of aqueous extract of the leaves of Hyptis suaveolens (L.) on acetaminophen induced hepatotoxicity in rabbits, Research Journal of Chemical Sciences 2011, 1(7), 85-88.

19. Mohammad N. Qureshi, Bhanudansh S. Kuchekar, Nadeem A. Logade, Majid A. Haleem, In-Vitro antioxidant and invivo hepatoprotective activity of Leucas ciliata leaves, Academy of Chemistry of Globe Publications 2010, 4(2), 124-130.

20. Rajeswari H, Vasuki R, Samudram P, Geetha A, Hepatoprotective action of ethanolic extracts of Melia azedarach Linn and Piper longum Linn and their combination on $\mathrm{CCL}_{4}$ induced hepatotoxicity in rats, Indian Journal of Experimental Biology 2011, 49, 276-281.

21. Shivananda Nayak B, Julien R.Marshall, Godwin Isitor, Andrew Adogwa, Hypoglycemic and hepatoprotective activity of fermented fruit juice of Morinda citrifolia (Noni) in diabetic rats, Evidence-Based Complementary and Alternative Medicine 2011, 1-5.

22. Samy M. Mohamed, Emad M. Hassan, Khaled A. Abd Elshafeek, Azza M. Mohamed, Investigation of flavonoidal constituents and hepatoprotective activity of Myoporum lactum, International Journal of Academic Research 2011, 3(3), 528-533.

23. Rupesh Kumar M, Pasumarthi Phaneendra, Surendra Bodhanapu, Fasalu Rahiman OM, Mohamed oiyas K, Tamizmani T, Antioxidant and Hepatoprotective activity of the aqueous extract of Myrtus communis (Myrtle) Linn leaves, Pharmacologyonline 2011, 1, 1083-1090.

24. Subash KR, Ramesh KS, Binoy Vargheese Charian Francis Britto, Jagan Rao N, Vijayakumar, Study of Hepatoprotective activity of Solanum nigrum and Cichorium intybus, International Journal of Pharmacology 2011, 7(4), 504-509. 